DOI: $10.5965 / 25946412222018047$

\title{
UTILIZAÇÃO DE MÍDIAS DIGITAIS COMO MEIO DE EDUCAÇÃO EM SAÚDE NO CONTEXTO DE EMERGÊNCIAS: EXTENSÃO UNIVERSITÁRIA
}

Ebenézer Pinto Bandeira Neto

Universidade Estadual do Ceará (UECE) ebenezerpbneto@gmail.com

Jonas Ramos Sales Universidade Estadual do Ceará (UECE) jonasramossales@gmail.com

Amanda Holanda Cardoso Maciel Universidade Estadual do Ceará (UECE) mandis.hcm@gmail.com

Gleiry Yuri Rodrigues Cardoso Universidade Estadual do Ceará (UECE) vurirodriguesc97@gmail.com

Daniel Barros Santos Correia Universidade Estadual do Ceará (UECE) danielbscorreia@gmail.com

Ana Flávia de Holanda Veloso Universidade Estadual do Ceará (UECE) afholandaveloso@gmail.com

Lídia Vieira do Espírito Santo Universidade Estadual do Ceará (UECE) lidiaesanto@gmail.com

Jôsivan Lima de Carvalho Universidade Estadual do Ceará (UECE) josivancarvalho2013@hotmail.com

João Gabriel Dias Barbosa Universidade Estadual do Ceará (UECE) joaogabrieldiasbarbosa@gmail.com 


\author{
Gislei Frota Aragão \\ Universidade Estadual do Ceará (UECE) \\ gislei.frota@uece.br \\ Bianca Rohsner Bezerra \\ Universidade Estadual do Ceará (UECE) \\ biancarohsner@gmail.com
}

\begin{abstract}
Resumo
Este artigo apresenta o projeto da Liga de Emergência do Curso de Medicina da Universidade Estadual do Ceará (UECE) com foco no uso de redes sociais como meio de propagar informações relativas a emergências cotidianas para uma população leiga, e na avaliação dessa metodologia adotada a fim de contribuir com a literatura. $\mathrm{O}$ texto tem por objetivo descrever a confecção e avaliação do projeto de extensão "Você sabe o que fazer quando se depara com uma situação de risco?". O projeto teve como resultados a confecção de banners abordando emergências cotidianas, e a divulgação destes em meios digitais. O crescente número de visualizações nas mídias e retorno positivo do público, o projeto cumpre o princípio da extensão universitária de levar informações da universidade à população, bem como permite, através do conhecimento, diminuir desfechos negativos em emergências.

Palavras chave: Redes sociais. Educação em saúde. Emergência.

\section{USE OF DIGITAL MEDIA AS A MEANS OF HEALTH EDUCATION IN THE EMERGENCY CONTEXT: UNIVERSITY EXTENSION}

\begin{abstract}
:
This article to present a project of the League of Emergency of the medicine course from the State University of Ceará (UECE) and is based on the use of social networks as a means to spread information related to daily emergencies to a lay population, and on the evaluation of this methodology in order to contribute to literature. The article describes and evaluates the preparation of the extension project "Do you know what to do when faced with a risk situation?". The project resulted in the making of banners addressing daily emergencies, in addition to the dissemination in digital media. With the increasing number of media views and positive feedback from the public, the project complies with the principle of university extension to bring information from the university to the population, as well as through knowledge, to reduce negative outcomes in emergencies.
\end{abstract}

Keywords: Social Network. Health education. Emergency.

\title{
UTILIZACIÓN DE MEDIOS DIGITALES COMO MEDIO DE EDUCACIÓN EN SALUD EN EL CONTEXTO DE EMERGENCIAS: EXTENSIÓN UNIVERSITARIA
}

\begin{abstract}
Resumen
Este artículo apresenta lo proyecto de la Liga de Emergencia, de curso de medicina, Universidad Estatal de Ceará (UECE) e y se basa en el uso de redes sociales como un medio de propagar informaciones relacionadas a emergencias cotidianas a una población laica y en la evaluación de esa metodología a fin de contribuir a la literatura. El artículo describe e evalúa la preparación del proyecto de extensión: "¿Sabe usted qué hacer ante una situación de riesgo. El proyecto resultó en la elaboración de banners que atender emergencias diarias, además de la difusión en medios digitales. Con el creciente número de medios de comunicación opiniones y comentarios positivos del público, el proyecto cumple con el principio de extensión universitaria para brindar información de la Universidad para la población, así como a través del conocimiento, para reducir los resultados negativos en situaciones de emergencia.
\end{abstract}

Palabras clave: Redes sociales. Educación para la salud. Emergencia.

Cidadania em Ação: Revista de Extensão e Cultura, Florianópolis (SC), v. 2, n.2, jul./dez. 2018. 


\section{INTRODUÇÃO}

A Emergência médica é definida, pelo conceito biomédico, como um processo de risco iminente de vida, que requer condutas de tratamento imediatas a fim de manter as funções vitais do paciente e evitar possíveis graves complicações. Nesse âmbito, podemos citar parada cardiovascular e traumatismo crânio encefálico como exemplos de situações de emergência médica (GIGLIO-JACQUEMOT, 2005).

A Previdência social no Brasil é um exemplo de setor associado a economia do País que sofre grande impacto gerado pela Emergência médica. Os gastos vinculados, principalmente, à superlotação de hospitais e ao elevado índice de incapacitância que aparece frente às sequelas das vítimas dessas situações, gera um ônus para o desenvolvimento do País. Além disso, é fundamental mencionar que a carga de estresse físico e mental a qual os médicos são submetidos nos grandes centros de emergência em virtude da imensa demanda impacta negativamente na vida destes profissionais. Esse cenário poderia ser minimizado por meio de práticas de prevenção de agravos, ainda não suficiente e eficazmente difundidas em nosso meio (POLL et al., 2008).

Por meio de práticas de educação em saúde é possível qualificar a população leiga para que esta seja capaz de agir de maneira mais adequada diante de diversas situações que oferecem risco de vida. E antes disso, é possível ainda reduzir a incidência de determinado agravo por meio de uma simples educação preventiva. O treinamento de uma comunidade que visa ao estímulo de habilidades em cuidados humanos é necessário para que se possa reduzir a enorme demanda existente, por exemplo, nos grandes centros de assistência emergencial. Para isso, é preciso a criação de um real e efetivo vínculo entre comunidade e profissional de saúde. A própria atividade de extensão universitária desenvolvida por estudantes da saúde tem papel fundamental em todo esse processo (PERETZ et al., 2012; PEREZ et al., 2006; IUAMOTO et al., 2012).

No âmbito da educação em saúde, uma estratégia atualmente adotada e que vem alcançando cada vez mais espaço nas práticas educativas, dentro e fora da Universidade, é o uso das Redes Sociais como forma de meio de comunicação e informação. Nesse aspecto, o público mais jovem, em especial de idades entre 18 e 30 anos, é o que mais se interessa por informações de saúde inseridas nas mídias digitais. Dessa forma, essa estratégia tem se mostrado extremamente eficaz em virtude do grande alcance populacional e da maior credibilidade que se pode dar ao conteúdo informativo nesse meio (PRYBUTOK e RYAN, 2015).

Cidadania em Ação: Revista de Extensão e Cultura, Florianópolis (SC), v. 2, n.2, jul./dez. 2018. 


\section{METODOLOGIA}

O projeto de extensão em tela foi desenvolvido por alunos de graduação do curso de Medicina da Universidade Estadual do Ceará (UECE), membros da Liga Acadêmica de Emergência da UECE (LEMERG), e supervisionado pelo professor orientador do projeto. $\mathrm{O}$ público-alvo foi a população leiga em geral, tanto interna quanto externa à universidade. $\mathrm{O}$ método utilizado foi a produção de banners informativos digitais sobre variados temas de emergências médicas.

Após a submissão, análise e aprovação do projeto, em janeiro de 2017 nas instâncias da Universidade, foi realizada uma reunião entre os membros da liga acadêmica e o professor orientador, durante a qual foram estabelecidos os temas que deveriam a ser contemplados e o cronograma de produção dos banners O período de vigência do projeto foi de março de 2017 até dezembro do mesmo ano.

No início de cada mês, com exceção de dezembro, no qual não houve produção, dois ou três membros da Liga foram escolhidos para elaborar um banner sobre o tema programado para cada mês. Dessa forma, todos os participantes colaboraram com o desenvolvimento do projeto. Inicialmente, a dupla ou o trio responsável realizava o levantamento bibliográfico sobre o tema, analisando e selecionando as informações mais relevantes para possibilizar ao público reconhecer a emergência em questão e, a partir disso, proceder de forma correta, sabendo o que deve ser feito e quais procedimentos são contraindicados. Destaca-se que foram utilizadas fontes bibliográficas confiáveis, isto é, fontes legitimadas como diretrizes (do Ministério da Saúde, Fiocruz e Sinitox, por exemplo), fontes bibliográficas reconhecidas na área a saber: Pronto-socorro: medicina de emergência. Herlon Saraiva Martins, Maria Cecília de Toledo Damasceno, Soraia Barakat Awada (Ed.).3. ed. Barueri (SP): Manole, 2013; e Emergências clínicas: abordagem prática. Herlon Saraiva Martins et al. 10. ed. rev. e atual. -- Barueri, SP: Manole, 2015. Deste título também foi utilizada a 11 ed. de 2016. No tocante a artigos científicos foram utilizados aqueles publicados por sociedades especializadas, como Sociedade Brasileira de Queimaduras e fascículos da Revista de Saúde Pública e Ciência \& Saúde.

A revisão bibliográfica possibilitou a fundamentação teórica dos banners, estes foram elaborados com informações adaptadas a uma linguagem mais acessível ao público leigo, de forma que o conhecimento fosse melhor incorporado.

A confecção propriamente dita dos banners foi realizada por meio dos programas Microsoft Office PowerPoint® e Adobe Photoshop®, realizando uma disposição gráfica dos conteúdos textuais e visuais de maneira atrativa para os leitores. Após serem finalizados, os Cidadania em Ação: Revista de Extensão e Cultura, Florianópolis (SC), v. 2, n.2, jul./dez. 2018. 
materiais eram encaminhados ao professor orientador, para que este pudesse emitir o seu parecer e sugerir possíveis alterações. Uma vez ajustados e aprovados pela coordenação do Projeto, os banners eram divulgados para o público alvo. Isso ocorreu, principalmente, na forma de mídias digitais, as quais foram disponibilizadas na página pública da LEMERG, na rede social Facebook $@$, e compartilhadas nos perfis pessoais dos membros da Liga, o que permitiu um maior alcance dos materiais.

Os dados coletados tiveram origem na própria plataforma digital utilizada para divulgação (Facebook@) e se referem ao número de visualizações de cada material, o que permitiu uma estimativa do tamanho do público alcançado. Após a coleta, foi utilizado o programa Microsoft Office Word ${ }^{\circledR}$ para análise dos dados e confecção de gráficos.

\section{RESULTADOS}

O projeto de extensão da Liga de Emergência do Curso de Medicina, da Universidade Estadual do Ceará (UECE) produziu nove banners no período de março a novembro de 2017 , um para cada mês. Os temas abordados respectivamente por mês foram: alívio de engasgo (março), queimaduras (abril), quedas (maio), intoxicações medicamentosas (junho), picadas por animais peçonhentos (julho), quedas (agosto), desmaios (setembro), infarto agudo do miocárdio (outubro) e cefaleia (novembro). Seguem alguns dos banners produzidos:

Figura 1:Exemplo de banner publicado com o tema "Infarto Agudo do Miocárdio"

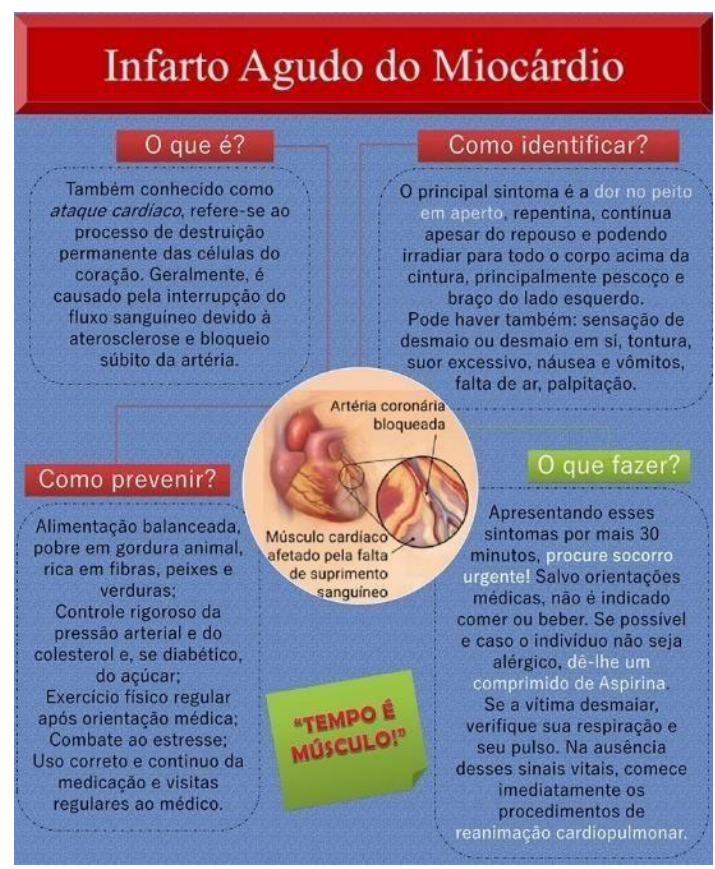

Fonte: Dados da Pesquisa (2017).

Cidadania em Ação: Revista de Extensão e Cultura, Florianópolis (SC), v. 2, n.2, jul./dez. 2018. 
Figura 2: Exemplo de banner publicado com o tema "Cefaleia"

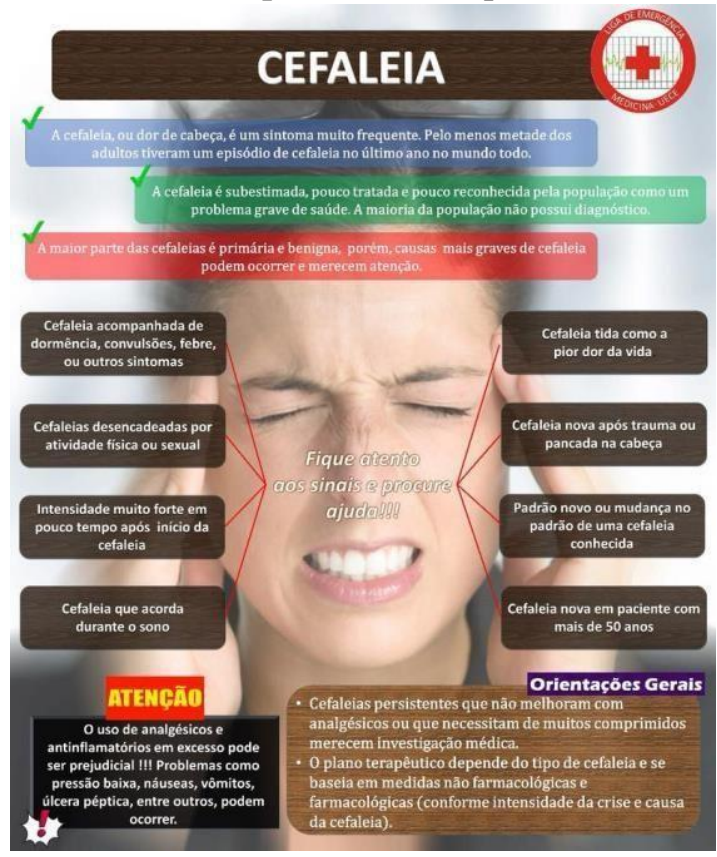

Fonte: Dados da Pesquisa (2017).

Figura 3: Exemplo de banner publicado com o tema "Desmaios"

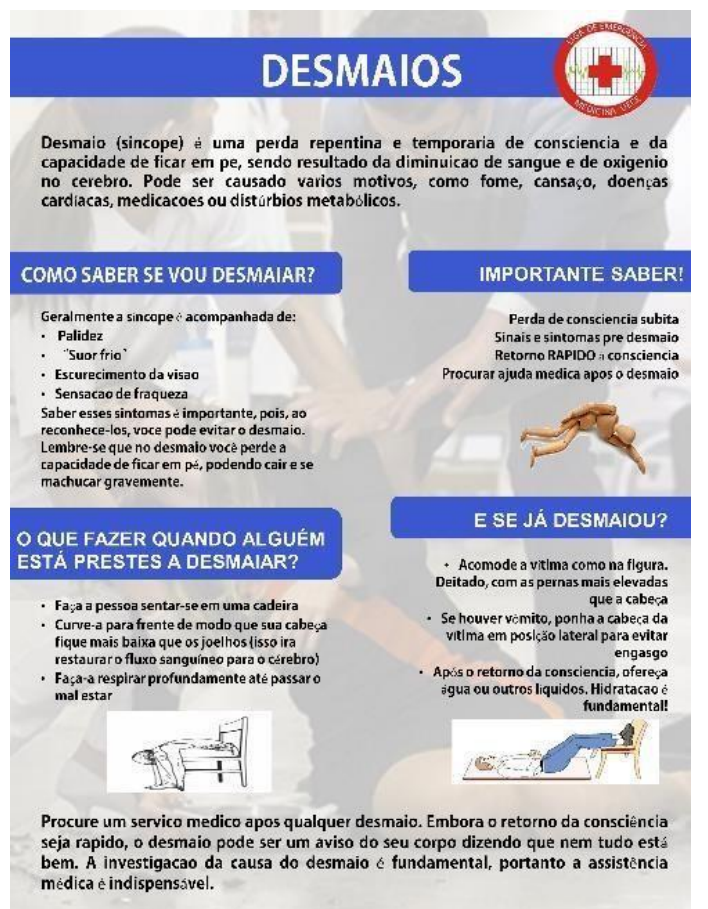

Fonte: Dados da Pesquisa (2017).

Cidadania em Ação: Revista de Extensão e Cultura, Florianópolis (SC), v. 2, n.2, jul./dez. 2018. 
Figura 4: Exemplo de banner publicado com o tema "Intoxicações Medicamentosas"

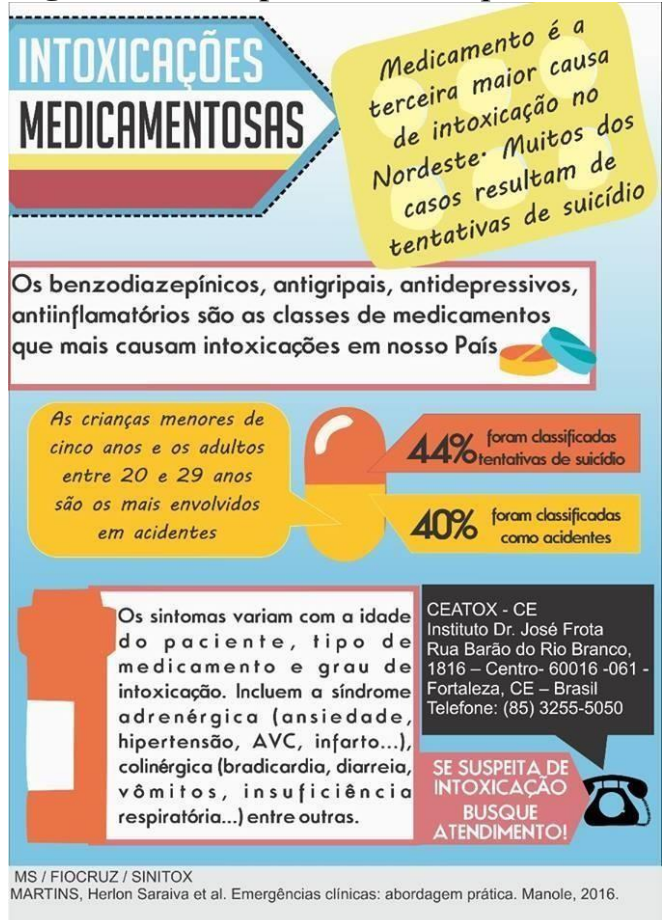

Fonte: Dados da Pesquisa (2017).

Figura 5: Exemplo de banner publicado com o tema "Queimaduras"

\section{QUEIMADURAS}

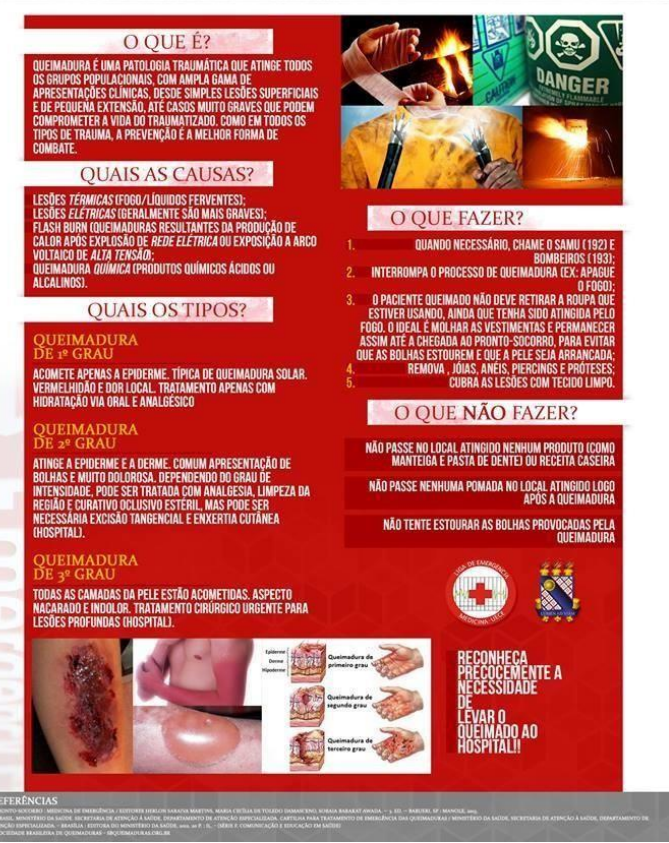

Fonte: Dados da Pesquisa (2017).

Cidadania em Ação: Revista de Extensão e Cultura, Florianópolis (SC), v. 2, n.2, jul./dez. 2018. 
Ao longo da realização do projeto, observou-se o acesso a um feedback das visualizações de cada banner digital, que nos foi fornecido pela plataforma (Facebook), o que nos permitiu ter um controle de qualidade sobre a produção dos banners. Os dados de acesso verificados foram de 254, 1.232, 534, 762, 1.708, 1.127, 2.366, 3.269 e 242 visualizações nos projetos publicados respectivamente nos meses de março, abril, maio, junho, julho, agosto, setembro, outubro e novembro.

Figura 6: Gráfico ilustrando número de visualizações de cada postagem ao longo do ano de 2017.

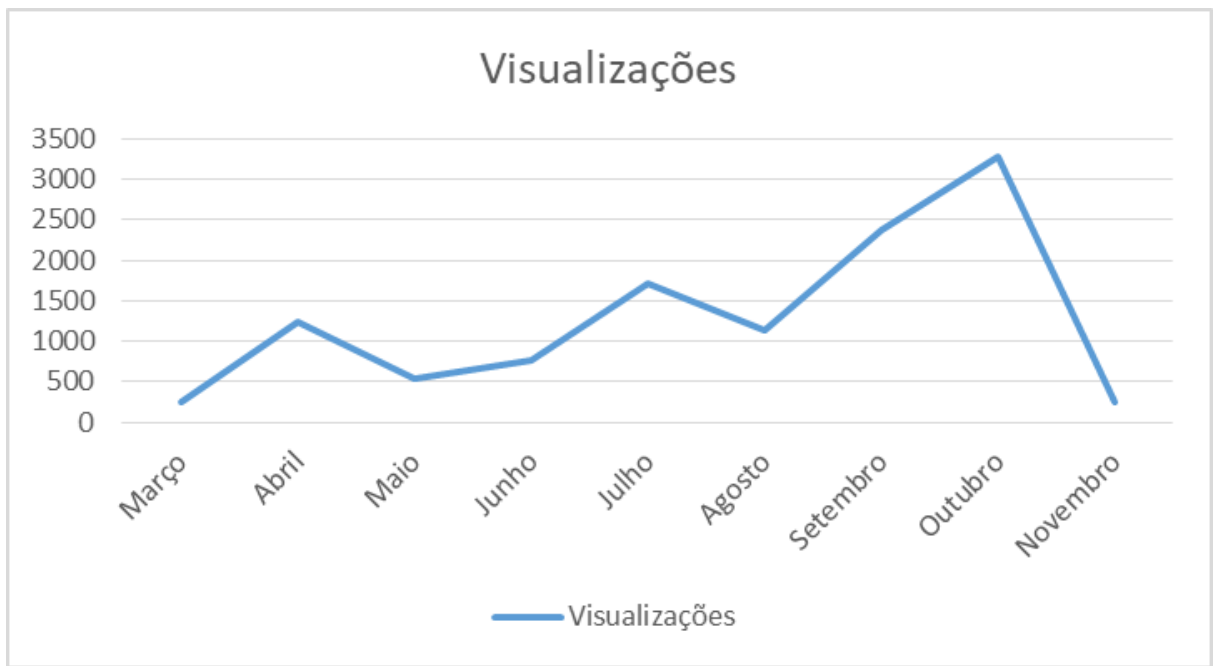

Fonte: Dados da pesquisa (2017).

Considera-se como positivo os resultados do projeto para despertar o interesse na produção científica que foi elaborada e disponibilizada em linguagem acessível, não apenas aos integrantes do grupo responsável, mas em pessoas que visualizaram o projeto e fizeram contatos com o grupo executor. Em outra ponta pode-se observar outro êxito alcançado por meio da realização do projeto foi a divulgação em si do grupo, ou melhor, da Universidade Estadual do Ceará visando a comunidade local para projetos futuros.

Entre as limitações do projeto consideram-se os custos das redes sociais colocam para uma disseminação em massa de conteúdo em suas plataformas, tornado a divulgação virtual pessoa a pessoa basicamente a única fonte de visualizações dos banners confeccionados. Apesar disso, o resultado do número de visualizações foi considerado positivo pelo grupo, devido ao pouco tempo de projeto e à nova metodologia aplicada no ano de 2017. Outra dificuldade pode-se apontar no desenvolvimento do projeto do projeto foi a pouca habilidade e experiência dos integrantes do grupo na produção gráfica dos materiais. Mas, em vez de permitirmos que isso constituísse um empecilho para a continuidade do projeto, tomamos isso Cidadania em Ação: Revista de Extensão e Cultura, Florianópolis (SC), v. 2, n.2, jul./dez. 2018. 
como estímulo para desenvolvermos tais habilidades, buscando ajuda em vídeos tutoriais e com pessoas experientes na área.

A questão educação em saúde relativa a procedimentos em casos de emergência apresenta pode ser observada em outros projetos encontrados na literatura. Fioruc et al. (2008). Trata-se de um estudo comparativo que utilizou questionários, para avaliar conhecimentos de primeiros socorros em professores e funcionários de escolas municipais da cidade local, antes e depois do treinamento oferecido pelo grupo. Os resultados bastante significativos obtendo na categoria de "procedimento correto" 42,8\% pré-treino e 90,5\% póstreino relativo a intervenção em caso de hemorragia externa; 42,8\% pré-teino e 79,4\% em póstreino relativo a convulsão; $33,3 \%$ pré e 84,1 pós, relativo a desmaio; $22,2 \%$ antes e $79,3 \%$ depois do treinamento, sobre sangramento nasal; dentre outros. Isso evidencia o extremo impacto que o fornecimento de informações acerca de como proceder em situações de emergência pode causar numa população leiga.

Outro estudo realizado por Lemos et al. (2012), semelhante ao apresentador anteriormente este com aplicação de questionários pré e pós curso, demonstrou uma melhora nos resultados dos participantes (professores e funcionários de uma escola particular) concernente aos temas convulsões, conduta após convulsões, engasgamento com adulto, engasgamento com criança, intoxicação, acidente com animais peçonhentos, reanimação cardio-pulmonar-cerebral, massagem cardíaca, queimaduras, condutas em queimaduras, cortes, hemorragias, afogamentos, condutas após afogamentos, entorses/fraturas e fraturas expostas. Isso coopera para a conclusão que obtivemos no parágrafo anterior.

Comparando nosso projeto a esses dois outros estudos acima expostos, identificamos que todos eles apresentaram objetivos semelhantes e abordaram pelo menos alguns temas em comum. No entanto, o fato de nosso projeto estar vinculado a uma plataforma digital e os outros dois serem cursos e questionários aplicados pessoalmente gerou diferenças de resultados que nos fazem refletir sobre o uso de mídias digitais como forma de propagar tal tipo de conhecimento. Os projetos realizados pessoalmente obtiveram a vantagem de ter como possibilidade uma ferramenta de feedback bastante segura, que evidencia o grau de conhecimento adquirido antes e depois da intervenção de educação em saúde.

Já no uso de uma ferramenta na plataforma digital, apesar de não ser tão garantido o resultado e consolidado como na aplicação de questionários pré e pós teste pessoalmente, isso nos fez refletir sobre a possibilidade de elaborar um questionário virtual a ser apresentado antes e depois da leitura dos banners, a ser utilizado em projetos futuros. Para além disso 
observamos que o nosso projeto obteve alcance de um número muito maior de pessoas, em razão a veiculação virtual. Isto é, obtivemos um alcance de mais de 3000 pessoas, comparando com os projetos supracitados estes tiveram o alcance de 63 e 21 pessoas, respectivamente. Concluído pode-se afirmar há vantagens e desvantagens de cada um dos tipos de projeto (presencial ou virtual), e nos faz entender a importância de ambos e sua complementariedade para promover uma melhor educação em saúde referente a intervenções em situações de emergência.

\section{CONCLUSÃO}

O projeto de extensão da Liga de Emergência do Curso de Medicina, da Universidade Estadual do Ceará (UECE) pode-se afirmar que alcançou os objetivos propostos enfrentando certas dificuldades inerentes ao desenvolvimento de atividades de extensão.

O objetivo do projeto vem sendo alcançado com sucesso, à medida que mais pessoas leigas têm acesso a informações cruciais sobre como identificar situações emergenciais e como proceder corretamente nesses casos. Além disso, outro foco atingido pelo nosso projeto é o de orientar na prevenção, tendo em vista que esta é uma das ferramentas de mais fácil execução para população em geral e que, usualmente, tem o maior potencial de salvar vidas. Assim, percebemos como podemos influenciar positivamente a população por meio do esclarecimento e também da desmistificação de diversos assuntos, tornando-a uma colaboradora ativa em situações emergenciais e, dessa maneira, ajudando a diminuir o número de desfechos negativos em tais situações.

Cidadania em Ação: Revista de Extensão e Cultura, Florianópolis (SC), v. 2, n.2, jul./dez. 2018. 


\section{REFERÊNCIAS}

FIORUC, B. E.; MOLINA, A. C.; VITTI JUNIOR, W.; LIMA, S. A. M. Educação em saúde: abordando primeiros socorros em escolas públicas no interior de São Paulo. Revista Eletrônica de Enfermagem, Goiânia, v. 10, n. 3, p. 695-702, set. 2008.

GIGLIO-JACQUEMOT, A. Definições de urgência e emergência: critérios e limitações. In: GIGLIO-JACQUEMOT, A. Urgências e emergências em saúde: perspectivas de profissionais e usuários. Rio de Janeiro: Fiocruz, 2005. p. 15-26.

IUAMOTO, L. R.; OIDE JUNIOR, M. S.; NAKAYAMA, J. T.; SHIOTUKI, R. S. Y.; KATO, J. M.; KUREBAYACHI, R.; SUZUKI, D. H. S.; LEE, A.; MORINAGA, C. V. Extensão Médica Acadêmica: um projeto da Faculdade de Medicina da Universidade de São Paulo para treinamento clínico e humanização do cuidado em saúde de alunos da medicina, nutrição e fisioterapia. Revista de Medicina, São Paulo, v. 91, n. 3, p. 194-197, 2012.

LEMOS, E. F. L.; NISIYAMA, A. L.; FARIAS, I. E. C.; MERCHAN-HAMMAN, E. Educação em saúde: a experiência de alunos de medicina no ensino em primeiros socorros. Participação: Revista de Extensão da Universidade de Brasília, Brasília, n.20, p. 35-42, jun. 2011.

PERETZ, P. J.; MATIZ, L. A.; FINDLEY, S.; LIZARDO, M.; EVANS, D.; MCCORD, M. Community health workers as drivers of a successful community-based disease management initiative. American Journal of Public Health, vol. 102, no. 8, p. 1443-6, 2012.

PEREZ, M.; MEJIA, M.; FINDLEY, S.; MARTINEZ, J. The impact of Community Health Worker Training and Programs in NYC. Journal of Health Care for the Poor and Underserved, vol. 17, no. 1, p. 26-43, 2006.

POLL, M. A.; LUNARDI, V. L.; LUNARDI FILHO, W. D. Atendimento em unidade de emergência: organização e implicações éticas. Acta Paulista de Enfermagem, São Paulo, v.21, n.3, p. 509-514, 2008.

PRYBUTOK, G.; RYAN, S. Social media: The Key to Health Information Access for 18- to 30-Year-Old College Students. CIN: Computers Informatics Nursing, vol. 33, no. 4, p. 132-141, apr. 2015. 